\title{
Acercamiento a las escuelas campesinas: entre voces de maestros*
}

APPROACHING RURAL SCHOOLS: AMONG THE VOICES OF TEACHERS

APROXIMAÇÃO ÀS ESCOLAS CAMPONESAS: ENTRE VOZES DE PROFESSORES

\author{
Ángela Mariel Loaiza Villalba / marielloaiza14@gmail.com
}

\section{Resumen}

Este artículo presenta la investigación realizada con el propósito de explorar la escuela campesina a través de las voces de sus maestras y maestros, para optar por el título de licenciadas en Educación Infantil de la Universidad Pedagógica Nacional. Con este fin realizamos viajes a los departamentos de Cundinamarca, Santander, Valle del Cauca y Caquetá, al encuentro con los maestros en sus propios territorios y se registraron las conversaciones, utilizando como opción metodológica la construcción de Historias de vida y las Narrativas sobre las escuelas y sus propias prácticas.

\section{Abstract}

This article presents a research that aimed to explore rural schools through the voices of their teachers, in order to obtain the title of BA in Early Childhood Education from the Universidad Pedagógica Nacional. For this purpose, we traveled to the departments of Cundinamarca, Santander, Valle del Cauca, and Caquetá, to meet with teachers in their own territories. Our conversations were recorded using as a methodological option the construction of life stories and narratives of teachers on schools and on their own practices.

\section{Resumo}

Este artigo apresenta a pesquisa realizada visando explorar a escola rural através das vozes de seus maestros e maestras, para obtenção do título de Licenciadas em Educação Infantil da Universidade Pedagógica Nacional. Com esse propósito, realizamos viagens aos departamentos de Cundinamarca, Santander, Valle del Cauca e Caquetá, para encontrar-nos com os maestros em seus próprios territórios. As conversações foram registradas, utilizando como opção metodológica a construção de Histórias de vida e as Narrativas sobre as escolas e suas próprias práticas.
Palabras clave

Campo, maestro, educación rural, escuela campesina.

\section{Keywords}

Countryside, teacher, rural education, rural school.

\section{Palavras chave}

Campo, maestro, educação rural, escola campesina.

\footnotetext{
El presente escrito es una síntesis de la investigación realizada por Mariel Loaiza, Juliana López y Mónica Jiménez para obtener el título de licenciadas en educación infantil, con la dirección de María del Pilar Unda Bernal. Las autoras contaron con la generosidad y los aportes de los siguientes maestros: Henry Miranda, maestro del Colegio Rural Quiba Alta, ubicado en la zona rural de Bogotá; Nereida Ávila, maestra de la escuela El Pórtico, ubicada en la vereda San Pedro, en Santander; Diego Vivas, de la escuela Sagrado Corazón de Jesús, ubicada en la vereda La Hondura, en el Valle del Cauca, y Edgar Gómez, coordinador del Colegio Rural Agroecológico Amazónico Buinaima, ubicado en la periferia del sur de Florencia, Caquetá.
}

Fecha de recepción: 10 de enero de 2016 / Fecha de aprobación: 29 de abril de 2016 


\section{(...) ;El futuro!, pues el futuro de ellos yo lo} sueño todo el tiempo, sueño a María como una maestra, sueño a Paola como una médica, porque yo sueño con ellos todas esas cosas y al que de pronto lo vea como que no quiere iyo lo pongo a soñar!

Diego Fernando Vivas (2015)

Después de darle y darle vueltas a la manera como debía escribir este artículo, intentado encontrar las palabras que describieran de la mejor manera el proyecto de investigación Acercamiento a las escuelas campesinas: entre voces de maestros, comprendí que lo más importante es que el lector conozca cada una de las narrativas y las experiencias vividas a través de la investigación. Y qué otra forma de hacerlo que adentrándonos en cada uno de los recorridos y los viajes que hicimos por cuatro departamentos de Colombia, para encontrarnos con cuatro maestros que llevan a cabo sus prácticas en el sector rural, y quienes nos dieron la oportunidad de conocer sus historias de vida expresando sus pensares, sus sentires, sus emociones y sus percepciones frente a la escuela rural colombiana. Por todo ello, este artículo se organiza en cuatro partes, que buscan contarle al lector las preguntas que nos hicimos, las vivencias en los viajes, los hallazgos y las conclusiones de la investigación.

\section{Parte I: antes de los encuentros}

Antes de emprender los viajes, hicimos una profundización en temas que nutrieron y aportaron herramientas conceptuales para la investigación, y entre los cuales se destacan tres. En primer lugar, un acercamiento a la "educación rural" en Colombia, a partir del estudio de autores como Orlando Fals Borda $(1961$; 1975) y Rodrigo Parra Sandoval (1996), lo cual nos permitió identificar la conexión entre la educación rural, los procesos de industrialización que se dieron en Colombia y la crítica al concepto de desarrollo. El interés del Estado en prestar más atención a la educación estuvo ligado, en sus inicios, a conseguir un desarrollo mercantil del campo; con tal perspectiva, se comenzó a enseñar a leer, a escribir y a utilizar las operaciones básicas de matemáticas a los campesinos.

A partir de ello, nos preguntamos: si bien la educación rural surgió como una educación pensada en beneficio de las dinámicas modernizantes que aprovechan el campo en beneficio del desarrollo y de la industrialización, ¿se identifican otras perspectivas, otras maneras de pensar el campo y al campesino desde la educación?

Por lo anterior, resaltamos en segundo lugar el trabajo de Diana Nocua en su tesis Prácticas Políticas del MST y el proceso Campesino y Popular de la Vega: hacia una mirada comparativa (2014). En esta se contrastan dos movimientos campesinos: uno en Brasil y otro en Colombia (Cauca), que han logrado, a través de muy interesantes procesos de organización y movilización, una ruptura con las políticas que pretenden homogenizar la educación desconociendo las particularidades de las distintas poblaciones. Son movimientos que han quebrado la idea de que el campo está ligado únicamente a la producción. Ambos movimientos son un ejemplo de educación pensada por y para el campesinado, replantean el concepto de educación rural y proponen, en cambio, profundizar en el de educación para el campo; este último encierra toda una serie de ideales, conceptos y posturas que defienden el derecho a una educación que responda a las reales potencialidades del campesinado, y se hizo fundamental explorar la manera como los maestros rurales manejan dichos conceptos.

En tercer lugar, fue fundamental explorar el surgimiento del maestro y el sitio que se le ha dado a través de la historia en Colombia, así como las críticas a los intentos de instrumentalizarlo, de convertirlo en simple transmisor de conocimientos y ejecutor de planes y programas determinados por el Estado. Pero el maestro se ha posicionado, gracias a los aportes del Movimiento Pedagógico, como un trabajador de la cultura y como productor de saber pedagógico, lo que llevó, entre otras cosas, a dimensionar su posicionamiento social y político. Esta tercera parte cerró una serie de cuestionamientos, preguntas e inquietudes, que llevamos a los encuentros con los maestros rurales.

\section{Parte II: encuentros con los maestros rurales}

Antes de conocer a estos cuatro maestros rurales, es importante mencionar que nuestra mayor expectativa en el presente trabajo era entender la voz del maestro como fundamental en la construcción de conocimiento sobre la escuela campesina, dado que es este quien la 
vive y la siente, quien la construye día tras día. Con esta investigación quisimos ir más allá de lo que se dice de los maestros "rurales", ir más allá de lo que se piensa de su labor, de su práctica, ir más allá de lo que dejan ver las políticas dirigidas hacia ellos. Quisimos escucharlos, darles valor a sus voces, saber lo que piensan, lo que hacen y lo que sienten frente a la escuela rural.

Con estos intereses como equipaje, nos dimos a la aventura de viajar a distintos territorios campesinos de Colombia, al encuentro con cuatro maestros en sus propios escenarios de práctica, aproximarnos a sus maneras de vivir y pensar la escuela campesina; como compañeras, elegimos las narrativas y las historias de vida. Escogimos cinco momentos para llevar a cabo esta experiencia: en primer lugar, el rastreo y la selección de los maestros a quienes conoceríamos; en segundo lugar, la realización de cuatro viajes a cuatro territorios campesinos de Colombia y los registros de las conversaciones sostenidas con sus maestros; en tercer lugar, la transcripción de las narrativas y de las historias de vida recogidas; un cuarto momento consistió en la tematización de las historias de vida en interacción con las herramientas de orden conceptual que nos Ilevaron a la realización de estos viajes, y, por último, los análisis y los hallazgos.

Utilizamos como herramientas para llevar a cabo esta investigación, por un lado, las historias de vida completas, las cuales delimitan la investigación a un tema, un asunto o un periodo de la vida del sujeto (Mckernan, 1999); por otro, las narrativas, debido a que nuestro interés se centró, principalmente, en sus vivencias y sus percepciones sobre la escuela rural, en su quehacer como maestros, en la concepción que tienen de la educación y de la escuela (particularmente, en los lugares donde se encuentran ejerciendo su profesión docente), en su relación con las comunidades y con las dinámicas diarias propias de cada territorio. Se hicieron entrevistas semiestructuradas como herramienta para la realización de las historias de vida, las cuales propiciaron espacios donde se logró interactuar directamente con esos maestros; así mismo, permitió recopilar experiencias significativas de ellos, las cuales giran en torno al ámbito educativo.

El primero de estos viajes se hizo a la zona rural de Bogotá, al Colegio Rural Quiba Alta, en Ciudad Bolí- var, el cual limita con los barrios de Quiba Baja, El Paraíso, Alpes y Guabal. Allí encontramos al maestro Henry Miranda Espitia, normalista y psicólogo social de profesión, con quien tuvimos la oportunidad de construir la historia de vida que denominamos "Entre el campo y la ciudad"; dicha historia refleja la tensión que enfrenta una escuela con dos realidades distintas: la realidad urbana y la realidad campesina, con niños y niñas que viven unos en el campo, y otros, en la ciudad.

Un par de correos y unas cuantas Ilamadas fueron suficientes para programar nuestro encuentro. Este tuvo lugar durante las primeras horas de un día frío del mes de febrero. La ansiedad y la incertidumbre de conocer a aquel maestro a quien nunca habíamos visto, nos invadía. Un buenos días, amable y cortés, bastó para reconocerlo; iba acompañado de unos guantes y un gorro, que hacían menos gélido su día. La conversación empezó: un par de preguntas, y el maestro Henry ya nos estaba contado su historia, sus experiencias, la manera como empezó su vida como educador y el gusto enorme que tiene por su profesión.

Tan maravillosa y agradable charla era necesaria terminarla visitando su escuela, acercándonos a esos proyectos que describía con tanto orgullo. Era necesario subir esa montaña envuelta por nubes espesas y frías y vivir la experiencia de conocer la escuela que había sido testigo de las numerosas vivencias relatadas.

Esta historia de vida nos dejó distintas percepciones frente a su práctica, de las cuales mencionaré, por el momento, dos: por un lado, los desafíos que se presentan en esta escuela, ubicada en uno de los bordes de la capital, debido a que son muy distintos los niños y las niñas que viven en el sector urbano de los que viven en el campo: sus condiciones de vida, las prácticas de la familia, las culturas, las sensibilidades y los modos de conocer. Este maestro nos habla de las dificultades que se le presentan en su escuela:

...tal vez si trabajáramos solamente con estudiantes del sector rural no habría tanto lío, o si trabajáramos solamente con estudiantes del sector urbano; pero el hecho de que haya esa mezcla, ahí empieza la primera problemática. H.M./Bogotá. 
Por otro lado, para el mismo maestro, las diferencias entre esta escuela periurbana y una escuela urbana son pocas en términos de accesibilidad a los recursos educativos, pero pueden ser grandes si se comparan con las escuelas ubicadas en otros sectores rurales:

... muy seguramente si vamos a hablar, de pronto, con un maestro y preguntarle qué es la educación rural por allá del Putumayo, del Caquetá, él sí puede tener una dimensión ¡muuuyy! diferente de lo que es educación rural, pero yo siento que aquí que estamos digamos, en el área periférica de Bogotá o periurbana... mmm la diferencia no es mucha. H.M./Bogotá.

Efectivamente, a estas diferencias se refieren los otros maestros que participaron en nuestro estudio; la percepción es que ni los recursos, ni las ayudas ni el apoyo del gobierno llegan de la misma manera en todas las escuelas colombianas.

Del "olvido" de las escuelas rurales nos habla la maestra Nereida Ávila, con quien nos encontramos en un segundo momento; un encuentro que se llevó a cabo en el departamento de Santander, a finales del mes de febrero:

Otra problemática es que muchas veces el gobierno olvida mucho las escuelas rurales, como el cuento, creen que porque uno está en el campo, pues, de pronto, no necesita mucho. N.A./Santander.

Antes de vernos, hablamos con ella, no más de tres veces; la lejanía de su escuela del casco urbano dificulta la comunicación. La maestra Nereida, licenciada en Educación básica primaria de la Universidad de Pamplona, labora en la escuela El Pórtico y refleja, con una voz fuerte y una mirada firme, el carácter singular de su población. Hemos denominado su historia de vida "Cueste lo que cueste".

La Escuela El Pórtico está ubicada en la vereda San Pedro, aproximadamente a 40 minutos de la capital santandereana. Llegar allí no fue fácil: por un lado, debido a la lejanía y a las carreteras extremadamente curvas; por otro, porque no teníamos idea alguna del lugar al que Ilegaríamos: todo lo que teníamos eran las indicaciones de Nereida, hechas por teléfono: Llegan al paradero de los paisas, ahí se bajan y cogen camino abajo. Luego de casi una hora de camino, a bordo de un bus que viajaba bajo un sol enorme y sofocante, Ilegamos a donde "los paisas"; lo de "camino abajo" indicaba andar bajo las ramas de los árboles, que pretendían abrazarnos. El silencio se hizo necesario para poder escuchar el ruido de nuestros pasos y el del viento cruzando por nuestro lado.

No íbamos solas, sino acompañadas por la incertidumbre y por el miedo, por la inseguridad y las ansias de encontrar una escuela que nos invitara a conocerla. Luego de 15 minutos de caminata, salieron de algún lugar cercano las risas y las voces de niños; estas nos devolvieron la seguridad, pues con ellas se asomaron un par de puertas abiertas, enormes, que nos invitaron a entrar en las historias y las memorias que abrazaban las paredes de la escuela.

Y así fue... nos adentramos en la escuela a través de la historia de vida de esta maestra, quien nos compartió la narrativa de su vida dejándonos conocerla y permitiéndonos descubrir nuevas cosas de la escuela rural campesina. Encontramos así que en esta escuela no sucede lo mismo que en las escuelas urbanas: los recursos y las ayudas prestadas por el gobierno son mínimas. Pero nunca lo dijo como un lamento; lo mencionó porque es debido a estas circunstancias - es decir, circunstancias difíciles- por lo que contempla otras más agradables y fascinantes, como la relación tan fuerte que se ha ido forjando entre la maestra y la comunidad de la vereda; parecería que los escasos recursos y el descuido por parte del Estado hacia la escuela han ido acercando tanto a la comunidad a todo cuanto sucede en la escuela, que han conseguido articular acciones y convertirlas en una sola para alejar la institución del deterioro y el abandono:

... tengo compañeros que laboran en la parte urbana, y hay una diferencia del cielo a la tierra. ¿Por qué? Porque en la parte urbana... hay menos colaboración por parte del padre de familia; en cambio, en el área rural la colaboración sí es bastante.... N. A./Santander.

En el mes de abril nos estábamos dirigiendo al departamento del Valle del Cauca, acompañadas por un grupo de maestros que participaban en la realización de 
viajes expedicionarios organizados por la Universidad Pedagógica Nacional, en convenio con el Instituto para la Investigación Educativa y el Desarrollo Pedagógico, IDEP. Íbamos en busca de la escuela Sagrado Corazón de Jesús, ubicada en la vereda La Hondura. A diferencia de los viajes anteriores, en este no teníamos definido a un maestro: íbamos en búsqueda de uno que quisiera compartirnos su experiencia. Entre las montañas nubladas de la Serranía de los Paraguas, nos acercamos a increíbles experiencias de maestros que en su cotidianidad están construyendo y reconstruyendo una práctica pedagógica profundamente arraigada y comprometida con su comunidad; entre ellos, Diego Fernando Vivas, quien labora en una de las sedes de esta escuela.

Esta historia de vida permitió que tomáramos conciencia de algo que teníamos presente, pero no había sido tangible hasta cuando no hablamos con el maestro Diego, y es la diferencia tan grande que existe entre un territorio y otro. Conocer esta experiencia, vivir con estos sujetos, y comprender las dinámicas de las escuelas ubicadas en esta vereda, es vivir una realidad completamente distinta de las que ya conocíamos. Es comprender lo que significa para un maestro tener que afrontar en su cotidianidad la guerra y la violencia que ha dejado el narcotráfico a su población:

esos niños que son los hijos de una violencia de 50 años, donde ellos lo que ven es la oportunidad, donde ellos lo que quieren es ser como el que ven que se bajó de la camioneta $4 \times 4$, con dos rubias y una botella de whisky en la mano, y en la otra, una pistola 9 milímetros y en los bolsillos Ilenos de dinero; es lo que ellos ven como una posibilidad, como oportunidad y "iyo quiero ser como él!". D.V./Valle del Cauca.

Luego de una conversación tan fructífera y sustancial con el maestro Diego, no queda otra cosa sino la percepción de un sujeto admirable y de una experiencia sorprendente. Escuchar las narraciones sobre una práctica pedagógica que pretende alejar a los niños y niñas, del narcotráfico y de la guerra que han dejado una historia de violencia en este territorio, es acercarse a un maestro increíble, a un sujeto conmovedor, a una historia deslumbrante, que hemos Ilamado "Construyendo caminos".

El mes de mayo se llenó de expectativas, al saber que haríamos nuestra próxima búsqueda en el departamento del Caquetá, una tierra que ha sufrido en gran medida por la violencia del país. El lector debe saber que fue, de los cuatro, el viaje más difícil, pues una de nosotras no pudo viajar, y en términos técnicos (grabadora, cámara) pasamos inconvenientes que por momentos hicieron que los ánimos se vieran casi perdidos. Ánimos que volvieron al escuchar por teléfono la voz del maestro Edgar Gómez, coordinador del Colegio Rural Agroecológico Amazónico Buinaima, ubicado en la periferia del sur de Florencia, y quien con su entusiasmo y su colaboración contribuyó a construir la historia de vida denominada "Explosión de sentimientos". Muchos de los relatos de este maestro estremecen, muchas de sus narraciones conmueven y aterran:

$¡$ ¡Ay! Hay muchas historias de cuando yo era profe de vereda que uno no quisiera recordar ni haber vivido (se queda en silencio un momento). La geografía del Caquetá es más o menos como un rectángulo, donde se atraviesan cosas dolorosas, como cuando atraviesas una hoja de papel; en los dos extremos están grupos armados, en un extremo del norte está la guerrilla, y en el extremo sur están los paramilitares, con su presencia en todos los caseríos (...) Era paradójico levantarse una mañana y mirar cómo en el muro de la casa y la escuela estaban los muertos colgados o los mensajes en el muro de muerte. E.G./Caquetá.

Este es un maestro que ha tenido que pasar por circunstancias duras, pero las ha afrontado con decisión, con una práctica pedagógica que irrumpe y resiste a todo cuanto quiere dañar a su comunidad, y da cuenta de los sueños que tienen los maestros rurales frente a su territorio, frente a su campo:

Yo sueño lo que sueña todo el mundo (suspira) de que haya una política de verdad en donde se respalde una organización agropecuaria, donde volver al campo sea económicamente productivo, donde haya también los recursos, los recursos necesarios para que se eduquen los chicos y las personas en general, tener acceso a los bienes y servicios: agua potable, Internet, ese tipo de cosas que son necesarias para tener un mejor nivel de vida, porque ser del campo no quiere decir ser marginado, sino que se garantice la dignidad humana. E.G./Caquetá. 


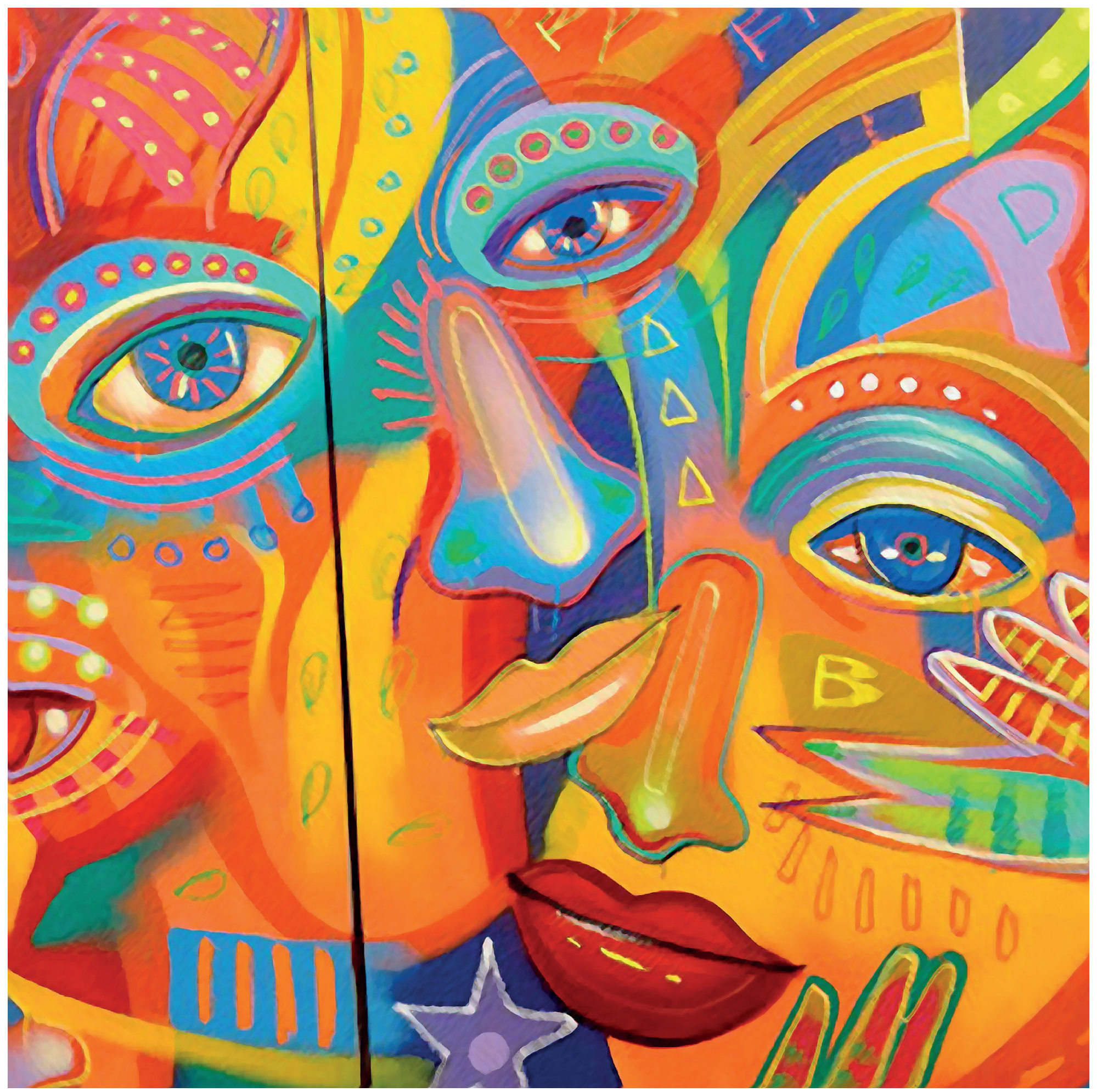

Artista: Guache 


\section{Parte III: hallazgos}

Los encuentros con cuatro maestros rurales y los registros de sus historias de vida y narrativas sobre la escuela permitieron hacer un conjunto de planteamientos sobre el hecho de ser maestro en el campo colombiano. A continuación, se mencionan las que se consideran los aportes más importantes para intentar comprender la escuela partiendo de las vivencias de los maestros rurales en Colombia.

\section{- Diversidad de ruralidades}

Encontramos, en primer lugar, que la ruralidad en Colombia no es homogénea, pues tiene variedad de territorios con condiciones, sujetos, historias, formas de vida, lógicas y dinámicas muy distintas. La ruralidad en Colombia se vive de maneras muy distintas, según las condiciones y las características de cada territorio. Por ello, es preciso hacer referencia a ruralidades.

- Diferencias entre la educación rural y la educación en la ciudad

Al explorar las condiciones de la educación en el campo, se encuentran referencias frecuentes a la distinción entre esta y la que se da en la ciudad: en primer lugar, por la distribución de los recursos, en tanto se privilegia a las grandes urbes y se tiende a dejar de lado a las poblaciones campesinas. $Y$, en segundo lugar, a las particularidades de las poblaciones campesinas, muy diferentes entre sí en cuanto a culturas, creencias, prácticas y costumbres. Encontramos también, a través de los relatos de estos cuatro maestros, que el Estado tiende a dejar en "abandono" a las escuelas campesinas, a centrar su atención y sus recursos en las ciudades. Los maestros rurales se refugian en otras organizaciones, donde buscan el apoyo necesario para mantener viva su escuela. Y también lo hacen en la comunidad, punto que se retoma a continuación.

- Maestros autónomos y relación entre la escuela y la comunidad

La manera como estos maestros viven la escuela del campo se mueve en una tensión: por un lado, sentimientos de tristeza y de soledad por las desigualdades sociales y sus consecuencias sobre la vida de las escuelas y de las poblaciones con las cuales trabajan; al mismo tiempo, cada historia de vida expresa la vitalidad y la decisión con que estos maestros enfrentan las adversidades, piensan, construyen y reconstruyen constantemente la escuela campesina.

- Maestros que transforman

Fue fundamental reconocer, a través de esta investigación, la manera como los maestros rurales se piensan a sí mismos, con autonomía; son maestros que valoran su quehacer, como motor de sus propias vidas. Son maestros que expresan una gran pasión por lo que hacen día a día en las escuelas campesinas.

- El campo como escenario de paz

Muchos de los relatos y las historias encontradas a través de la investigación dan cuenta del conflicto que ha tenido que pasar el campo colombiano: guerra, violencia, narcotráfico, etc. Sin embargo, estos cuatro maestros afirman, con orgullo, ser campesinos, y son maestros que conciben el campo como escenario de paz, que debe ser respetado. ¿Por qué? Percibimos que, más allá de cuanto haya debido sufrir el campo, sus actores están comprometidos con construir una paz; por ello, sus prácticas pedagógicas dejan entrever acciones, pensamientos y sueños para el campo colombiano.

Indudablemente, cada viaje permitió que experimentáramos nuevas sensaciones, nuevos olores, nuevos paisajes. Cada viaje trajo consigo a personas admirables, pero, sobre todo, cada viaje aportó significativamente a la construcción de una nueva mirada de las escuelas campesinas. Una mirada construida a partir de las voces de los maestros, quienes nos abren a historias, a anécdotas, a pensares, a sentires y a perspectivas frente a su escuela, frente a su labor, frente a su territorio.

\section{Parte IV: conclusiones}

La riqueza que existe en nuestro país respecto a las costumbres, las formas de pensar el territorio y las dinámicas que se viven en él permite reconocer que la ruralidad se vive de maneras muy distintas, y que no podemos hablar de una sola ruralidad, pues las condiciones de los campesinos son muy diversas; por ello, es preciso referirnos, más bien, a ruralidades, diversas y numerosas. 
Los valores, las culturas, los sueños y las propuestas de vida de las poblaciones campesinas se convierten en claves para la definición de las propuestas pedagógicas de los maestros que aportaron sus historias de vida y sus narrativas en el presente estudio. No se encuentran huellas de los enfoques desarrollistas o de instrumentalización del campo con fines economicistas en sus discursos. Y aun cuando este tema se conecta con la diferenciación entre educación rural y educación para el campo, no se hallaron referencias al respecto en los registros obtenidos en el estudio.

El reconocimiento de las particularidades y de las condiciones de vida de cada territorio requiere redefinir las políticas educativas y una distribución más equitativa de los recursos educativos para las escuelas. Pero consideramos que lo más importante es dar a conocer y ampliar el debate sobre los modos de pensar el campo y los aportes de los campesinos: tal vez, la más interesante contribución de los movimientos campesinos de los movimientos campesinos citados en nuestro estudio.

En relación con el tema de la formación de maestros, se hace necesario que los diferentes programas académicos vinculen la ruralidad como eje; que contribuyan a reconocer las dinámicas y las circunstancias a las cuales se enfrentan los maestros en esos territorios, para así generar propuestas que tengan en cuenta y no pierdan de vista las especificidades de dichos territorios.

Podemos visualizar la educación que se da en el campo con diferentes perspectivas: una, ligada a las políticas y las leyes dirigidas hacia ella, y a los fines que se pretenden por medio de ella desde el Gobierno y el Estado; otra, ligada a la forma como se hace escuela campesina desde sus principales actores: los maestros; nos enorgullece presentar una aproximación a esta última, como un modo de oponerse a las finalidades que buscan la tecnificación o la modernización del campo por medio de la escuela, y que rompe con los esquemas y las concepciones de la educación rural.

Esta investigación nos permitió darle lugar al maestro, como sujeto que porta y produce saber a través de sus prácticas educativas, desde sus escuelas y las particularidades de los territorios.

\section{Referencias}

Arenas, J. (1985). Cese al fuego. Una historia política de las FARC. Bogotá, Colombia: Editorial Oveja Negra.

Caballero, E. (1974). Los campesinos. Bogotá, Colombia: ANTARES.

Fals Borda, O. (1961). Campesinos de los Andes. Bogotá, Colombia: IQUEIMA.

Fals Borda, O. (1975). Historia de la cuestión agraria en Colombia. Bogotá, Colombia: ıQueima.

Giroux, H. (1990). Alfabetización, escritura y política de sufragio. Barcelona: Paidós Ibérica.

Martínez, A., Unda, M. del P. y Mejía, M. R. (2002). El itinerario del maestro: de portador a productor de saber pedagógico. En Veinte años del Movimiento Pedagógico. Bogotá, Colombia: Tercer Milenio.

Molano, A. (1990). Siguiendo el corte. Relatos de guerras y de tierras. Bogotá, Colombia: Áncora.

Nocua, D. (2014). Prácticas Políticas del mst y el proceso Campesino y Popular de la Vega: Hacia una mirada comparativa. Trabajo de grado, Maestría en Educación Comunitaria, Interculturalidad y Ambiente, Universidad Pedagógica Nacional de Colombia.

Parra, R. (1996). Escuela y modernidad en Colombia: Tomo II la escuela rural. Bogotá, Colombia: Tercer Mundo Editores.

Programa de las Naciones Unidas para el Desarrollo (PNUD) (2011). Colombia rural, razones para la esperanza. Bogotá, Colombia: PNUD.

Rodríguez, A. (2002). 20 años, 1982-2002, del Movimiento Pedagógico, entre mitos y realidades. Bogotá, Colombia: Cooperativa Editorial Magisterio.

Salgado, C. (2002). Los campesinos imaginados. Bogotá, Colombia: ILSA 176.

Zamora, F. (2005). Huellas y búsquedas. Una semblanza de las maestras y maestros rurales colombianos. Bogotá, Colombia: Fundación Universitaria Monserrate - Fundación Santa María

Zamora, F. (2010). ¿Qué es lo rural de la educación rural? El sentido y alcances de la categoría educación rural. Medellín, Colombia: Tercer Congreso Nacional de Educación Rural (ponencia).

Webgrafía

Blanco, M. (2011). Investigación narrativa: una forma de generación de conocimientos. México: Distrito Federal. Recuperado de: http://www.redalyc.org/articulo.oa?id=59521370007

Bolívar, A. y Domingo J. (2006). La investigación biográfica y narrativa en Iberoamérica: Campos de desarrollo y estado actual. Recuperado de: http://www.qualitative-research.net/index.php/ fqs/article/view/161/357

Díaz, J. (1998-2005). La campaña de cultura aldeana (1934-1936) en la historiografía de la educación colombiana. Recuperado de: http://www.pedagogica.edu.co/storage/rce/articulos/rce3839_11balan.pdf

Fajardo, D. (2010). Tierra, poder político y reformas agraria y rural. Recuperado de: http://www.kus.uu.se/CF/Cuaderno_01.pdf. 
Federicci, C., Mockus, A., Charum, J., Granés, J., Castro, M. C., Guerrero, B. y Hernández, C. (1984). Límites del cientificismo en educación. Recuperado de: http://www.pedagogica.edu.co/ storage/rce/articulos/14_08pole.pdf

González, P. (coord.). (1985). Historia política de los campesinos latinoamericanos. Recuperado de: https://books.google.com. co/books?id=Jb8cQBwLvGgC\&pg=PA38\&lpg=PA38\&dq=Oq uist $\% 2 C+1975 \&$ source $=$ bl\&ots $=$ scTqWtxG2J\&sig=xs5C5Rk8 TpYh1uQPIQJ9GI8I1X8\&hl=179 es\&sa=X\&ved=0CCwQ6AE wA2oVChMlq6HMo46lyAIV0TweCh3anAqc \#v=onepage\&q $=$ Oquist $\% 2 \mathrm{C} \% 201975 \& \mathrm{f}=$ false

Informe General Centro Nacional de Memoria Histórica capítulo II (2012). Las herencias de la vieja violencia: Barbarie, exclusión y enemigos internos. Recuperado de: http://escolapau.uab.cat/ img/ programas/procesos/12anuarie.pdf

Jiménez, T. (2007-2008). El desplazamiento humano en Colombia: ¿Disminuye o Aumenta? Recuperado de: http://www.observatori.org/documents/Desplazamiento_humano_en_Colombia. pdf

Mckernan, J. (1999). Investigación, acción y curriculum. Madrid: Morata. Disponible en: http://eduneg.net/generaciondeteoria/ files/Mckernan $\% 20$ Investigacion-accion $\% 20 y \% 20$ curriculum. pdf

Ministerio de Educación Nacional, República de Colombia (1994). Ley General de Educación. En Ministerio de Educación Nacional [en línea], 8 de febrero. Recuperado de: http://www. mineducacion.gov.co/1621/articles-124745_archivo_pdf9.pdf [2014, 9 de octubre]

Núñez, J. (2004). Los saberes campesinos: implicaciones para una educación rural, Recuperado de: http://www.scielo.org.ve/ scielo.php?pid=S1316-00872004000200003\&script=sci_arttext

Pueblo colombiano pa' la mesa (2015). Recuperado de: https://www. pazfarc-ep.org/index.php/cronologia
Quijano, A. (2000). La colonialidad del saber: eurocentrismo y ciencias sociales. Perspectivas latinoamericanas. Buenos Aires, Argentina: CLACSO.

Reyes, Y. (2015). Retrato de la desigualdad. Recuperado de: http:// www.eltiempo.com/opinion/columnistas/retrato-de-la-desigualdad-yolanda-reyes-columnista-el-tiempo/16244680

Saldarriaga, O. (2003). Del oficio del maestro: prácticas y teorías de la pedagogía moderna en Colombia. Recuperado de: http://www.academia.edu/1255794/Del_oficio_de_maestro_Prácticas_y_teorías_de_la_pedagogía_moderna_en_ColombiaSilva, S. (2008). Movimiento campesino colombiano: historia y lucha. Colombia. Recuperado de: http://www.prensarural.org/ spip/spip.php?article1289

Suárez, H. (2015). Carta de Estanislao Zuleta a los maestros. Necesitamos maestros que nos identifiquen con los fracasados. Recuperado de: http://www.las2orillas.co/carta-de-estanislaozuleta-los-maestros/

Supelano, B. (2011). Tejido social campesino. En: Despertar campesino, capítulo 5. Recuperado de: http://www.fundaexpresion. org/PDF/despertar\%20campesino\%20C-5.pdf

Vasco, C. (1985). Límites de la crítica al cientificismo en educación. Recuperado de: http://www.pedagogica.edu.co/storage/rce/ articulos/16_08pole.pdf

Zuluaga, O. (1986). Historia epistemológica de la pedagogía o Historia del saber pedagógico. Recuperado de: https:// books.google.com.co/books? id=qNbh64WGU7EC\&p $\mathrm{g}=$ PA55\&lpg=PA55\&dq="Historia + epistemológica + de + la +pedagogía+o+Historia+del+saber+pedagógico" \&source $=$ bl\&ots $=$ Lc_qdXxzsY\&sig $=i 8$ PwmLuSWRh2 ckmT0zkJTqRtA\&hl $=$ es-419\&sa $=X \&$ redir_esc $=y \# v=$ onepage $\& q=\% E 2 \% 8$ 0\%9CHistoria\%20epistemol\%C3\%B3gica\%20de\%20la $\% 20$ pedagog $\%$ C3\%ADa\%20o\%20Historia $\% 20$ del\%20saber $\% 20$ pedag $\%$ C3\%B3gico $\%$ E2\%80\%9D\&f=false 


\section{Diálogo del conocimiento}

Hay que valorar positivamente de este escrito varios puntos: 1) el hecho de darles voz a los maestros rurales para referirse a lo que hacen profesionalmente y a las condiciones en las cuales lo hacen. Buena parte del escaso reconocimiento que en Colombia le otorgamos a la educación rural proviene de que se habla de esta educación desde parámetros urbanos, lo que casi siempre conduce a identificar lo rural con una "colección de carencias", sin mayor identidad;2) subrayar, desde el inicio de la experiencia, la "diversidad de ruralidades" presentes en nuestra geografía nacional, diversidad que no siempre es percibida por los propios maestros desde sus territorios, sino por quien hace, justamente, dicha tarea de investigación en el terreno; 3) la emergencia de ciertas "potencialidades" propias del contexto campesino (por oposición a la "colección de carencias"), aunque no se adentra en ellas; 4) el agradable estilo de narración, que se complementa con oportunas citas tomadas del trabajo con los maestros rurales visitados.

En cuanto al primer punto, este trabajo nos recuerda los importantes aportes que en tal sentido les debemos a Rodrigo Parra S., su hermano Francisco Parra S., a Gonzalo Cataño y, a mediados del siglo xx, a Dieter Pass.

En cuanto a lo segundo, cabe destacar la importancia de profundizar sobre esta diversidad de ruralidades en Colombia, aunque en el texto se dan solo algunas puntadas en ese sentido. Por su lado, las "potencialidades" del contexto rural se nos presentan aquí más como un postulado que como un hallazgo.

Para terminar, advierto en el texto una debilidad (que es frecuente en trabajos de esta naturaleza, elaborados por educadores), en cuanto a que se nos anuncia y se hace hincapié en algo llamado "pedagogía de lo rural", en este caso como un hallazgo, pero no se ofrecen elementos que permitan llenar de contenido tan importante categoría. Sería de mucho interés que posteriormente la autora dedicara un artículo específico a desarrollar este punto. Las instituciones formadoras de maestros se podrían alimentar de ello, cuando de preparar educadores para lo rural se trata, cosa que hasta el momento representa una notable falencia en los currículos de tales instituciones.

Como lector, me quedó la sensación de un final algo precipitado en el escrito; de pronto, porque estaba esperando cierto desarrollo de algunos de los puntos antes mencionados (en especial, del último).

Fernando Zamora Guzmán 